\title{
The Challenge of Complex Dynamics for Theoretical Models of Cardiac Activity*
}

\author{
ARKADY KUNYSZ, ALVIN SHRIER and LEON GLASS ${ }^{\dagger}$ \\ Department of Physiology, McGill University, 3655 Drummond Street, Montreal, Quebec, Canada H3G 1 Y6
}

(Received 29 November 1996)

\begin{abstract}
Electrical stimulation of excitable cardiac tissue results in a variety of rhythms similar to those clinically recorded. Simplified nonlinear equations and ionic models give complementary descriptions of the dynamics in cardiac preparations. Nonlinear models often capture the essential dynamical features of the experimental observations but do not properly describe the underlying ionic mechanisms. Ionic models are descriptive, complex, and despite their general success in reproducing the cardiac action potential, seldom undergo extensive testing against experimentally determined rhythms. We review work from our group devoted to the modeling of cardiac rhythms, including nonlinear models and the Shrier-Clay ionic model of electrical activity in embryonic chick heart cell aggregates. Since sustained stimulation may have long lasting consequences on the intrinsic excitability of the tissue, we also discuss recent advances in modeling the stimulation history-dependent aspects of pacemaking.
\end{abstract}

Keywords: phase resetting, entrainment, ionic models, nonlinear dynamics

\section{INTRODUCTION}

Two classes of theoretical formulations have been used to model cardiac dynamics. The nonlinear dynamics approach, initiated by van der Pol (van der Pol and van der Mark, 1929) develops simplified mathematical models of cardiac activity. These models often admit detailed mathematical analysis, and may be broadly applicable to a range of similar behaviors in different preparations. Examples of simplified nonlinear models for cardiac activity include (Guevara et al., 1981; Guevara and
Glass, 1982; Winfree, 1987; Chialvo, 1990; Vinet and Roberge, 1994; Sun et al., 1995). These simplified models do not provide sufficient insight into the ionic mechanisms governing the onset of the experimentally or clinically observed cardiac rhythms. The second theoretical approach, ionic modeling, is directly based on the detailed description of the individual mechanisms underlying cellular electrical activity. These models originated with the Hodgkin-Huxley formulation of the ionic basis of the action potential in the squid axon, and have subsequently been extended to cardiac

\footnotetext{
${ }^{*}$ Based on a presentation in Workshop on Modelling the Heart, Edinburgh, Scotland, October 1994

${ }^{\dagger}$ Tel: 514-398-4338, Fax: 514-398-7452, e-mail: glass@cnd.mcg:1l.ca
} 
tissues (McAllister et al., 1975; Noble and Noble, 1984; Shrier and Clay, 1986; Luo and Rudy, 1991; Wilders et al., 1991). Ionic models are developed based primarily on voltage and patch-clamp data. Usually, the goal of the modeling is to describe the cardiac action potential. However, in some cases, ionic models have been subjected to a variety of stimulation protocols with a view towards comparing dynamics with experiment (Dexter et al., 1989; Liu et al., 1993; Rudy and Luo, 1993; Dokos et al., 1993; Kowtha et al., 1994; Beaumont et al., 1995) Clay et al., 1990.

Since the response of biological oscillators to external perturbation is often a very sensitive function of the timing of the stimulus, it is critical to ensure that ionic models not only reproduce the action potential but are capable of adequately reproducing experimental dynamics under a variety of stimulation protocols. We review results from our group obtained during single or sustained electrical stimulation of embryonic chick heart cell aggregates. The experimental data is compared with numerical simulations of the Shrier-Clay ionic model of activity in this preparation. Since stimulation history may affect the response of the preparation to subsequent stimuli, we have begun to incorporate time dependent effects into our theoretical descriptions of cardiac dynamics.

\section{METHODS}

Embryonic chick heart cell aggregates were prepared using methods described previously (DeHaan, 1967; Shrier and Clay, 1986). Briefly, heart cell aggregates comprised of cells from the atria of seven-day-old embryonic chicks were grown in tissue culture. The transmembrane potential was measured using a microelectrode filled with $3 \mathrm{M} \mathrm{KCl}$. The same microelectrode was also used to electrically stimulate the preparations. Temperature was maintained at $36 \pm 1{ }^{\circ} \mathrm{C}$. The stimulation protocols were remotely controlled with a $\mathrm{PC}$-based programmable stimulator (Alembic Software, Montreal, Canada). The voltage waveforms were recorded on a FM instrumentation recorder (Hewlett-Packard 3964
A), at a tape speed of 3 ips, for subsequent off-line analysis.

The stimulation protocols consisted of: (1) phase resetting: a single depolarizing stimulus (duration of $20 \mathrm{msec}$ ) was injected every 10 spontaneous action potentials, at increasing phases of the cycle: (2) periodic stimulation: 50 periodically spaced electrical stimuli of constant amplitude were delivered at different stimulation frequencies; (3) overdrive suppression: sustained periodic stimulation was applied using different train durations, stimulus frequencies and intensities.

Numerical simulations were carried out by using the Shrier-Clay ionic model of activity (Shrier and Clay, 1986; Clay et al., 1990; Kowtha et al., 1994). This model consists of five components: $I_{\mathrm{Na}}$, the fast sodium current responsible for the rapid action potential upstroke; $I_{\mathrm{Ca}}$, the calcium current playing a role in the late part of the upstroke of the action potential and in maintaining membrane depolarization during the plateau phase of the action potential; $I_{K_{\mathrm{S}}}$, the time-dependent outward current involved in the initial phase of repolarization; $I_{K_{\mathrm{r}}}$, the timedependent inwardly rectifying current responsible for the late phase of repolarization; and $I_{\mathrm{b}}$ a threecomponent backward current underlying depolarization during phase 4 of the cycle. Numerical simulations were carried out using a variable step Euler integration method (Moore and Ramon, 1974) for the transmembrane potential and a Rush-Larsen (Rush and Larsen, 1978) iteration method for the gating parameters.

\section{RESULTS}

\section{Phase Resetting}

Phase resetting is an important concept in the study of biological oscillators, and refers to a rapid readjustment of the phase of the cycle in response to some external stimulus (Winfree, 1980; Glass and Mackey, 1988). In oscillating tissue from nerve or heart, the stimulus is generally electrical. The response of a single cell, or a group of cells, to external stimulation depends upon the intensity, 
polarity, and duration of the applied electrical current, and on the timing of the stimulus with respect to the intrinsic cycle (Perkel et al., 1964; Guevara, Glass and Shrier, 1981; Clay et al., 1984; Guevara et al., 1986). The phase resetting curve (PRC) is obtained by delivering isolated stimuli at different phases of the cycle and measuring the resulting perturbed cycle length (normalized to control) as a function of the phase of the stimulus. Under the assumption that the stimulus does not alter the intrinsic properties of the oscillation but only resets the phase of the cycle, the PRC can be used to predict the response of the tissue to periodic stimulation protocols (Perkel et al., 1964; Pavlidis, 1973; Guevara, Glass and Shrier, 1981; Guevara and Glass, 1982; Glass et al., 1984, 1986;
Zeng et al., 1990; Kowtha et al., 1994). Since phase resetting is important in interpreting experimentally observed dynamics, it is essential that ionic models aimed at describing the electrical activity of spontaneously active cardiac tissue undergo extensive testing against experimental phase resetting data.

In embryonic chick heart cell aggregates, a phase resetting experiment is performed by injecting $20 \mathrm{msec}$ isolated depolarizing current pulses, every 10 spontaneous action potentials, at increasing phases of the cycle. One such phase resetting curve, obtained from a $130 \mu \mathrm{m}$ atrial aggregate (stimulus amplitude of $30 \mathrm{nA}$, control cycle length $T_{0}=400 \mathrm{msec}$ ) is shown in Figure 1 (open circles), superimposed on a numerical simulation of the Shrier-Clay ionic model of activity (stars,

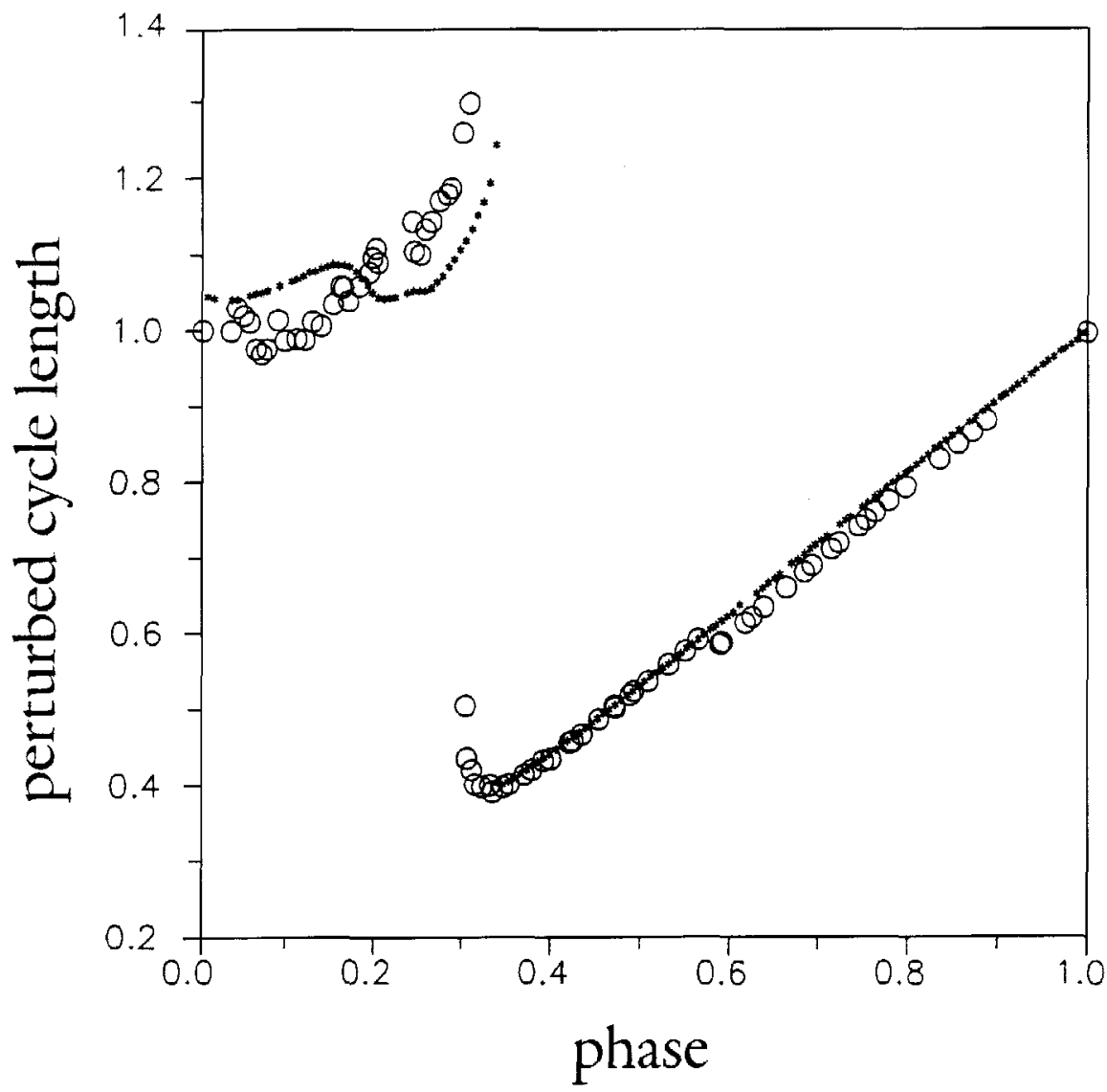

FIGURE 1 Phase resetting in embryonic chick heart cell aggregates and in the Shrier-Clay ionic model. Experimental data (symbols, $130 \mu \mathrm{m}$ aggregate, control cycle length of $400 \mathrm{msec}$, stimulus intensity $=30 \mathrm{nA}$ ) is superimposed on numerical simulation of the ionic model (stimulus amplitude $=50 \mathrm{nA}$, control cycle length $=380 \mathrm{msec}$ ). 
amplitude: $50 \mathrm{nA}, T_{0}=380 \mathrm{msec}$ ). Since pulse intensity must be scaled to the size of the preparation, the amplitude of the stimulus in the numerical simulation was chosen to reproduce the general shape of the experimental PRC. Short stimulus phases correspond to delayed action potentials. Stimuli delivered later in the cycle elicit premature action potentials. The simulations of the ionic model closely mimic the experimental results. A more detailed study of phase resetting in the Shrier-Clay equations (including the dependence upon stimulus intensity) can be found in Kowtha et al. (1994).

In the Shrier-Clay ionic model, the $I_{\mathrm{K}_{\mathrm{r}}}$ and $I_{\mathrm{Na}}$ components are the primary ionic mechanisms responsible for phase resetting, as illustrated in Figure 2 (amplitude is $40 \mathrm{nA}$, as in Figure 1) for stimulus phases corresponding to the vicinity of the transition between phase delay and phase advance in the PCR. In both panels, the time courses of $I_{\mathrm{K}_{\mathrm{r}}}$ and $I_{\mathrm{Na}}$ are shown below the simulated voltage traces. A stimulus injected shortly after the time of maximum diastolic polarization effectively augments the amplitude of $I_{\mathrm{K}_{\mathrm{r}}}$. Since an important fraction of $I_{\mathrm{K}_{\mathrm{r}}}$ channels remain open for approximately $100 \mathrm{msec}$ after maximum diastolic potential, MDP, a depolarizing stimulus of sufficient intensity increases the driving force on the residual $I_{\mathrm{K}_{\mathrm{T}}}$ by moving the membrane potential away from $E_{k}$. Since the $I_{\mathrm{K}_{\mathrm{r}}}$ time constant increases with depolarization (from $-90 \mathrm{mV}$ to $-60 \mathrm{mV}$ ), the deactivation of the residual $I_{\mathrm{K}_{\mathrm{r}}}$ channels is slower than under control conditions. Moreover, the depolarizing pulse also causes premature activation of $I_{\mathrm{Na}}$. The advance or delay of the next action potential depends upon the precise timing of the stimulus. At early stimulus phases, $I_{\mathrm{K}_{\mathrm{r}}}$ dominates over $I_{\mathrm{Na}}$ resulting in a phase delay. If the pulse is applied only slightly later, the combination of a slightly greater deactivation of $I_{\mathrm{K}_{r}}$ and of an increase in $I_{\mathrm{Na}}$ (the membrane depolarizes closer to the $I_{\mathrm{Na}}$ threshold) gives rise to a premature action potential.

The above simulations were carried out with a control cycle length of $380 \mathrm{msec}$, which corresponds to the low range of experimental intrinsic cycle lengths, typically found for very small aggregates. On average, the control cycle length of embryonic chick atrial heart cell aggregates is between 450 and $650 \mathrm{msec}$ (at $37^{\circ} \mathrm{C}$ ). Despite these various experimental cycle lengths, the corresponding phase resetting curves (for comparable stimulus intensities) show a great degree of resemblance (Clay et al., 1984, Guevara et al., 1986, Zeng et al., 1990). For slowly beating aggregates, the transition between phase delay and phase advance is usually shifted to smaller phase values. However, the general shape of the phase resetting curve and parameters such as the maximum phase delay are only slightly affected
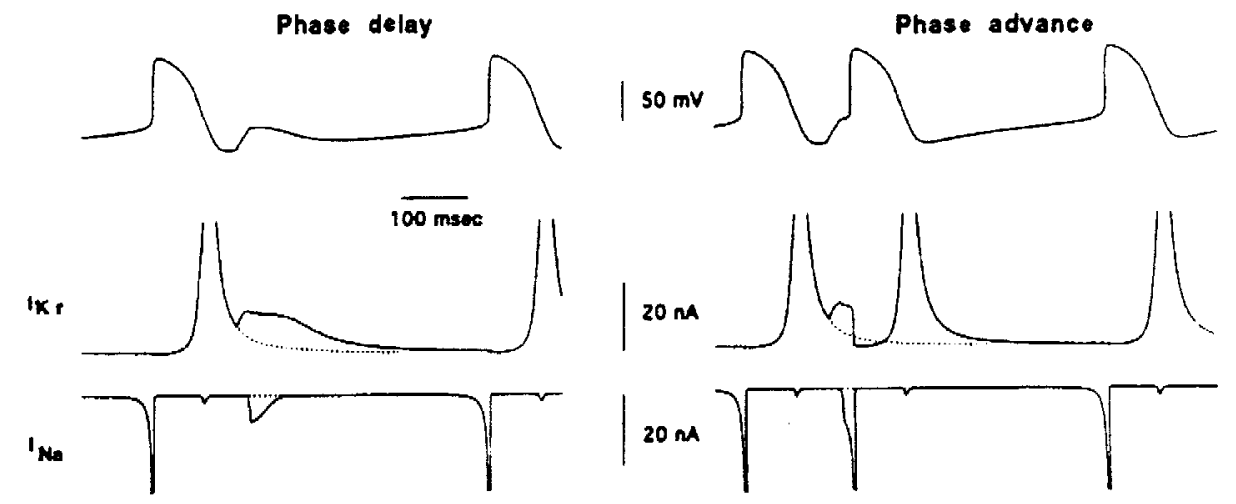

FIGURE 2 Ionic mechanisms of phase delay and advance in the Shrier and Clay (1986) model, as described in the text. A $40 \mathrm{nA}$ current pulse was applied in the model shortly after MDP, as indicated in the voltage waveform in each panel. The time of the pulse in the right-hand panel was $1 \mathrm{msec}$ later than in the left hand panel. $I_{\mathrm{k}_{\mathrm{r}}}$ and $I_{\mathrm{Na}}$ components are shown below the voltage waveform in each panel. The dashed lines illustrate the behavior of these components in the absence of a current pulse. From Kowtha et al. (1994), by permission. 
by changes in control cycle length. This observation is not well reproduced in the simulations of the Shrier-Clay equations. Although the cycle length of the Shrier-Clay equations can be adjusted by rescaling one of the components of the background current $\left(I_{\mathrm{b}_{1}}\right)$, such changes strongly influence the appearance of the phase resetting curves. These effects are not properly understood and represent one of the weaknesses of the present formulation of the Shrier-Clay ionic model of electrical activity.

\section{Phase Locking}

In the healthy heart, the cardiac rhythm is entirely synchronized to the beating of the sino-atrial node: there is $1: 1$ entrainment between the sinus rhythm and the rest of the myocardium. Many dysrhythmias are characterized by a loss of the 1:1 entrainment with the sinus rhythm. This loss of $1: 1$ entrainment normally appears as a result of one of the following causes: (1) block of conduction of the electrical impulse; (2) competition between the dominant and a subsidiary pacemaker; (3) a re-entrant circuit. The abnormal rhythm can be either regular or irregular. Experimental protocols using sustained periodic stimulation are designed to study the dynamics that arise during competition between pacemakers. This section summarizes results from our group on the effects of periodic stimulation of cardiac aggregates and in theoretical models of the aggregates (Guevara, Glass and Shrier, 1981; Glass et al., 1984; Zeng et al., 1990; Kowtha et al., 1994).

Figure 3(A) illustrates some of the rhythms observed during sustained periodic stimulation of embryonic chick heart cell aggregates. A small $100 \mu \mathrm{m}$ aggregate $\left(T_{0}=300 \mathrm{msec}\right)$ was stimulated with trains of 50 stimuli (amplitude $=25 \mathrm{nA}$ ) separated by rest intervals of 30 seconds. A control voltage trace appears on top. The corresponding simulated entrainment rhythms are shown in Figure 3(B). With stimulus frequencies close to the intrinsic beating rate of the preparation, there is 1:1 entrainment between the stimulator and the aggregate For stimulus periods significantly smaller than the control cycle length, more complex
$N: M$ (stimuli : action potentials) locking patterns are found $(N>M)$. The locking ratio $(M / N)$ typically decreases with frequency in a stepwise fashion, often called the 'devil's staircase' (Glass and Mackey, 1988). The details of the structure of the phase locking zones are dependent upon stimulus intensity. Under certain conditions, 1:1 entrainment can also be maintained with stimulus frequencies slightly lower than the intrinsic beating rate of the preparation. In some cases, irregular or chaotic rhythms are also found.

Figure 4 illustrates the ionic mechanisms underlying the development of a 5:4 rhythm. The top voltage trace is the same as in Figure $3(\mathrm{~B})$. The time courses of $I_{\mathrm{K}_{\mathrm{r}}}$ and $I_{\mathrm{Na}}$ are shown below. During $N: M(N>M)$ entrainment, neither $I_{\mathrm{K}_{\mathrm{r}}}$ nor $I_{\mathrm{Na}}$ dominate after each pulse. Arrows $a$ indicates the increase in $I_{\mathrm{K}_{\mathrm{r}}}$ described above, after the first current pulse depolarizes the membrane away from $E_{k}$, as well as the corresponding increase in $I_{\mathrm{Na}}$. In this case, the domination of $I_{\mathrm{Na}}$ over $I_{\mathrm{K}_{\mathrm{r}}}$ gives rise to a premature action potential. The next pulses $(\boldsymbol{b}-\boldsymbol{c})$ fall increasingly closer to MDP, leaving less time for $I_{\mathrm{K}_{\mathrm{r}}}$ to deactivate before stimulation. However, $I_{\mathrm{Na}}$ still dominates and premature action potentials are elicited. Pulse $\boldsymbol{d}$ falls even earlier with respect to MDP. Here, $I_{\mathrm{K}_{\mathrm{r}}}$ is larger than $I_{\mathrm{Na}}$ : there is a skipped beat. Since $I_{K_{\mathrm{r}}}$ is mostly inactivated at the time of the next stimulus, an action potential is evoked and the sequence resumes. Finally, arrows $f$ indicate the slight reactivation of $I_{\mathrm{Na}}$ during the repolarization of the action potential. Note that, by increasing the stimulation frequency, a lesser number of action potentials is necessary before the domination of $I_{\mathrm{Kr}}$ results in a skipped activation.

The rhythms observed during periodic stimulation depend upon the stimulus frequency and intensity (Figure 5). The abscissa is the period of stimulation normalized to control cycle length; the ordinate is the amplitude of the stimulus. The symbols correspond to various experimental locking patterns, as determined by visual inspection of traces from seven different aggregates. The solid lines demarcate the boundaries of the phase locking zones determined by iteration of the phase resetting curves obtained 

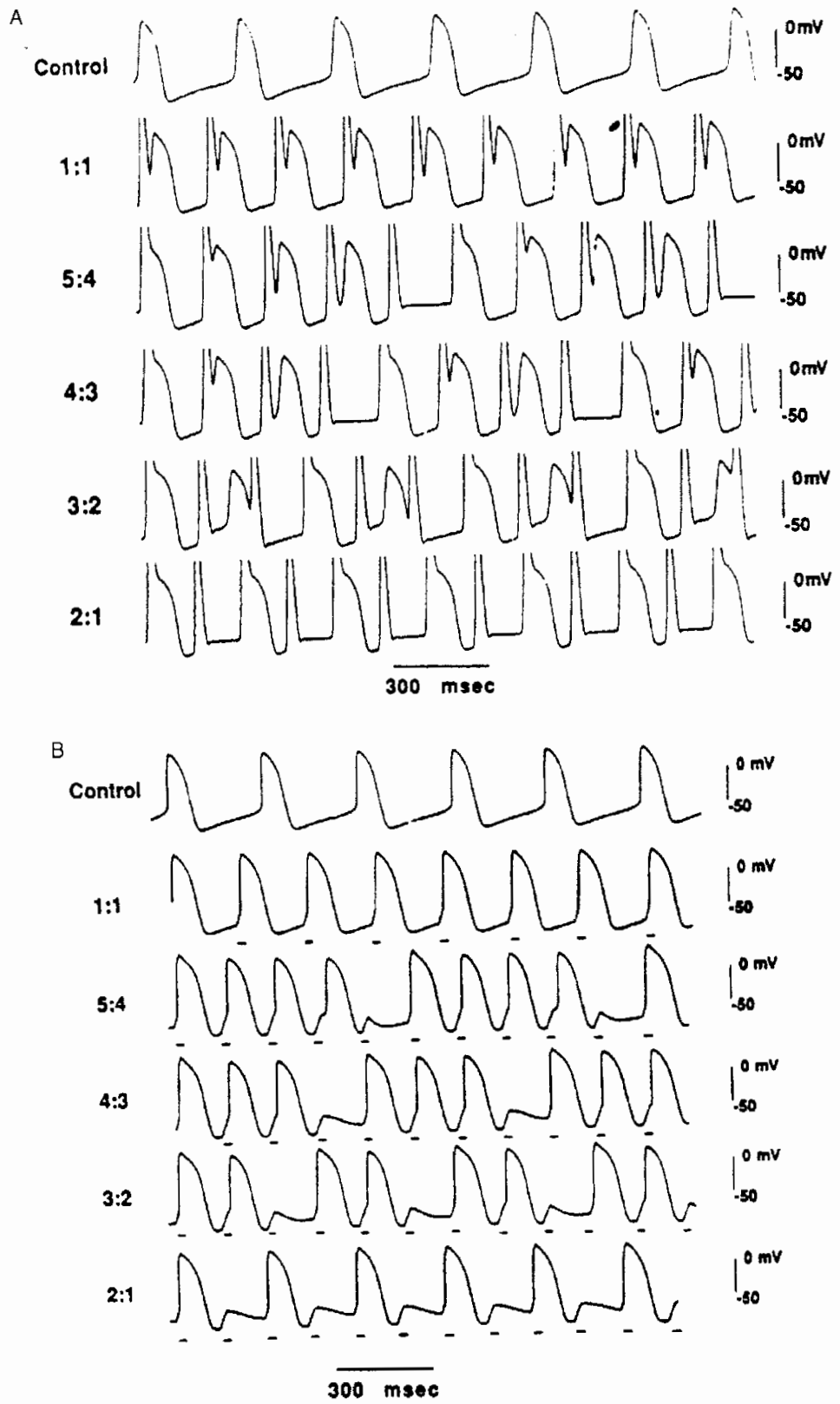

FIGURE 3 Comparison of experimental and simulated phase locked rhythms. Panel A: Experimentally observed rhythms intermediate to $1: 1$ and $2: 1$ phase locking. Top panel illustrates control spontaneous activity from a preparation different from that illustrated in Figure $1\left(T_{0}=300 \mathrm{msec}\right)$. Shown below the control trace are $1: 1,5: 4,4: 3,3: 2$, and $2: 1$ phase locking for $i_{\text {puise }}=25 \mathrm{nA}$, and $t_{\mathrm{S}}=220,200,190,180$, and $160 \mathrm{msec}$, respectively. Panel $\mathrm{B}$ : Rhythms intermediate to $1: 1$ and $2: 1$ phase locking in the Shrier and Clay $(1986,1990)$ model. The $I_{b 1}$ parameter was adjusted so that $T_{0}=300 \mathrm{msec}$. These results were obtained with $i_{\text {pulse }}=25 \mathrm{nA}$ and $t_{\mathrm{s}}=200,184,178,160$ and $150 \mathrm{msec}$, respectively. From Clay et al. (1990), by permission. 

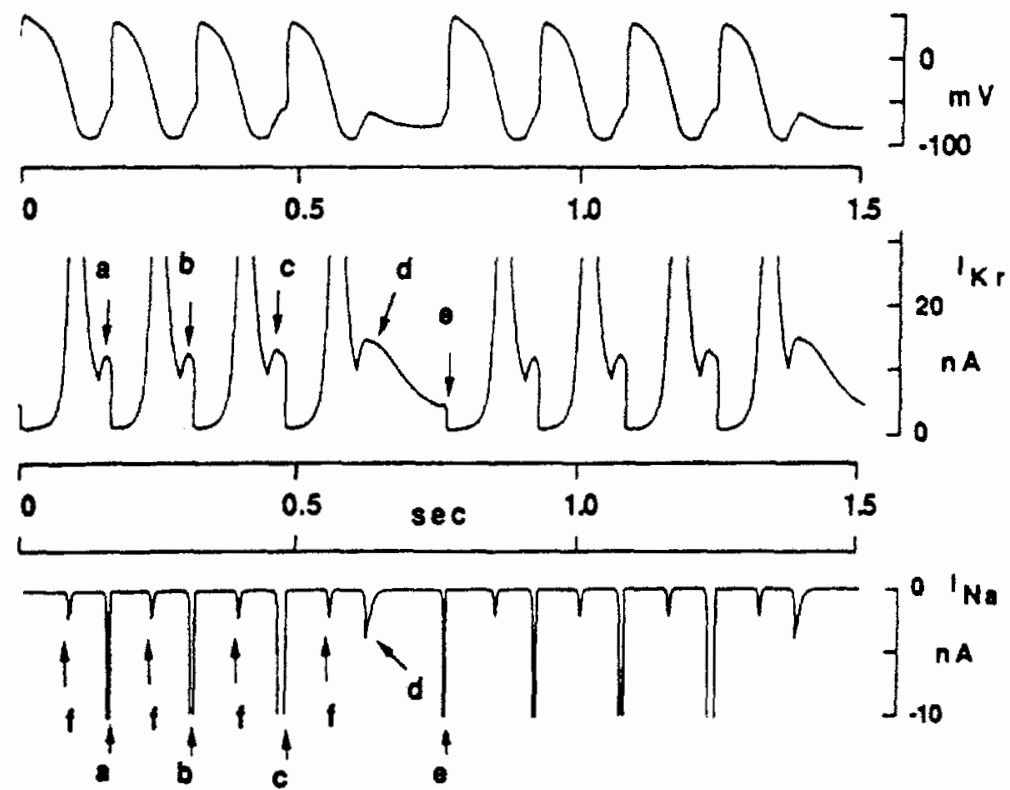

FIGURE 4 Ionic basis of 5:4 entrainment in the Shrier and Clay (1986) model as described in the text. The bottom two panels illustrate the behavior of $I_{\mathrm{k}_{\mathrm{r}}}$ and $I_{\mathrm{Na}}$ in this pattern. From Kowtha et al. (1994), by permission.

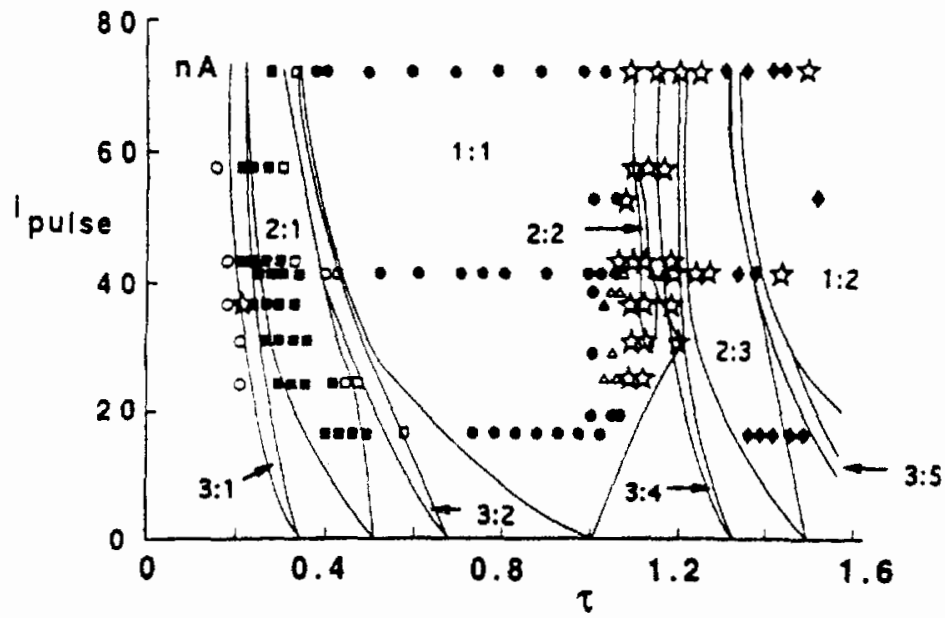

FIGURE 5 Phase locking zones. The various symbols illustrate different phase locked rhythms, as determined from visual inspection of experimental recordings from seven different aggregates. These results have been normalized according to the configuration of the PRC, so that they all correspond to a $200 \mu \mathrm{m}$ diameter aggregate. The symbols represent $3: 1\left({ }^{\circ}\right) ; 2: 1(\square) ; 3: 2(\square) ; 1: 1(\bullet) ; 2: 2(\Delta)$; $2: 3($ ); and irregular rhythms $(*)$, respectively. The boundaries of various zones in the ionic model (as indicated by the hand-drawn lines) were determined from the PRCs using the iterative approach described in the text. The $I_{\mathrm{b} 1}$ component in the Shrier and Clay (1986, 1990) model was set so that $T_{0}=366 \mathrm{msec}$. From Kowtha et al. (1994), by permission.

by numerical simulation of the Shrier-Clay ionic model. The method used to iterate the phase resetting curves relies on the assumptions described in the previous section. Let $\phi_{i}$ denote the phases of the $i$ th current pulses, $T_{0}$ the intrinsic cycle length, and $T\left(\phi_{i}\right)$ the perturbed cycle length induced by a stimulus delivered at phase $\phi_{i}$. Under the assumption that the stimulus leads to an instantaneous transition from one phase on the cycle to another, the relationship between successive phases of the 
stimulus can be written as a difference equation

$$
\phi_{i+1}=1+\phi_{i}-\frac{T\left(\phi_{i}\right)}{T_{0}}+\tau,(\operatorname{mol} 1)
$$

$\tau$ is the stimulus period normalized to the intrinsic cycle length. Given an initial phase $\phi_{0}$, this equation can be iterated to determine the dynamics (Perkel et al., 1964; Pavlidis, 1973; Guevara, Glass and Shrier, 1981).

In Figure 5, the largest zone corresponds to 1:1 locking. Other zones occupy much smaller regions of parameter space. The translational symmetry arises from equation (1) (Guevara and Glass, 1982). Both period-doubled rhythms (i.e. 2:2, 4:4 ...) and chaotic dynamics (stippled zones) are observed in the experiments as well as well as the theoretical models (Guevara et al., 1981, 1982; Glass et al. 1984, 1986; Zeng et al., 1990).

\section{Overdrive Suppression}

Overdrive suppression is the transient lengthening of the intrinsic cycle length following sustained stimulation at a rate faster than the control frequency of the preparation (Vasalle, 1970, 1977; Courtney and Sokolove, 1979; Pelleg et al., 1980; Musso and Vassalle, 1982; Gilmour et al., 1987; Greenberg and Vassalle, 1990; Zeng et al., 1991; Prinsze, 1993; Kunysz et al., 1995). Overdrive is generally believed to be linked to the activation of a hyperpolarizing ionic current, electrogenic pump, or exchanger - the precise mechanism might be species and tissue specific. In embryonic chick heart cell aggregates, overdrive suppression is markedly reduced by the application of micromolar concentrations of ouabain, a blocker of the sodium-potassium pump (Pelleg et al., 1980).

We briefly summarize studies of overdrive suppression from our group in embryonic chick heart cell aggregates (Zeng et al., 1991; Kunysz et al., 1995). A representative tracing of overdrive suppression in embryonic chick atrial heart cell aggregates $(150 \mu \mathrm{m}$ aggregate, control cycle length of $550 \mathrm{msec}$ ) is shown in Figure 6. Five spontaneous action potentials are shown at the beginning of the voltage trace. Following the application of 50 rapid stimuli during which each stimulus evokes an action potential, the first spontaneous interbeat interval is approximately double the spontaneous cycle length. The intrinsic cycle length then slowly decreases towards the control value. This decay of the cycle length as a function of time can be described by a single or double exponential function (Zeng et al., 1991), with time constants of the order of 2 and 20 seconds.

The magnitude of overdrive suppression induced by periodic stimulation with $1: 1$ entrainment is a function of the drive duration. Since the application of a long, rapid train of stimuli results in a marked prolongation in the cycle length, Zeng et al. (1991) ascribed the previously described evolution of rhythms to the time-dependent modulation
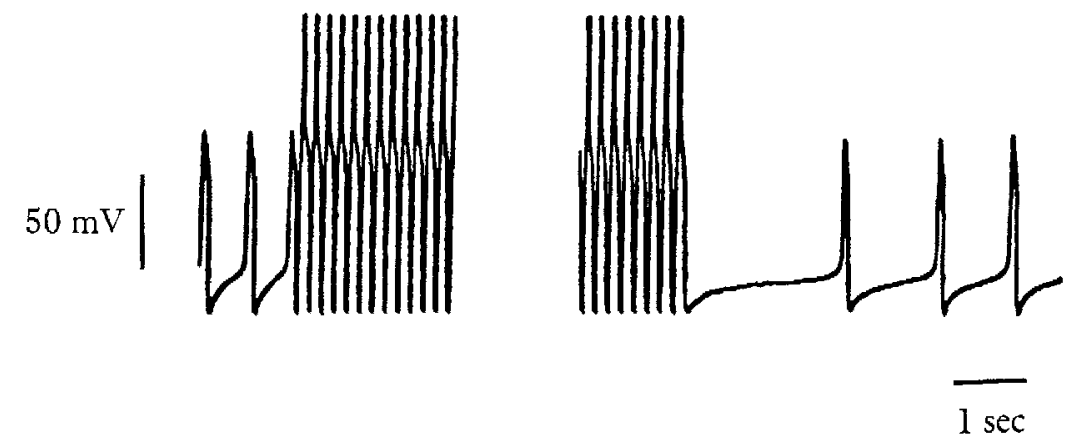

FIGURE 6 Overdrive suppression in embryonic chick heart cell aggregates. An atrial aggregate of diameter $180 \mu \mathrm{m}$ and control cycle length $470 \mathrm{msec}$ was stimulated with trains of 50 pulses of fixed period ( $145 \mathrm{msec}, 1: 1$ entrainment). Stimuli appear as the off-scale artefacts. For clarity, not all the pulses are shown. Adapted from Kunysz et al. (1995), by permission. 
A
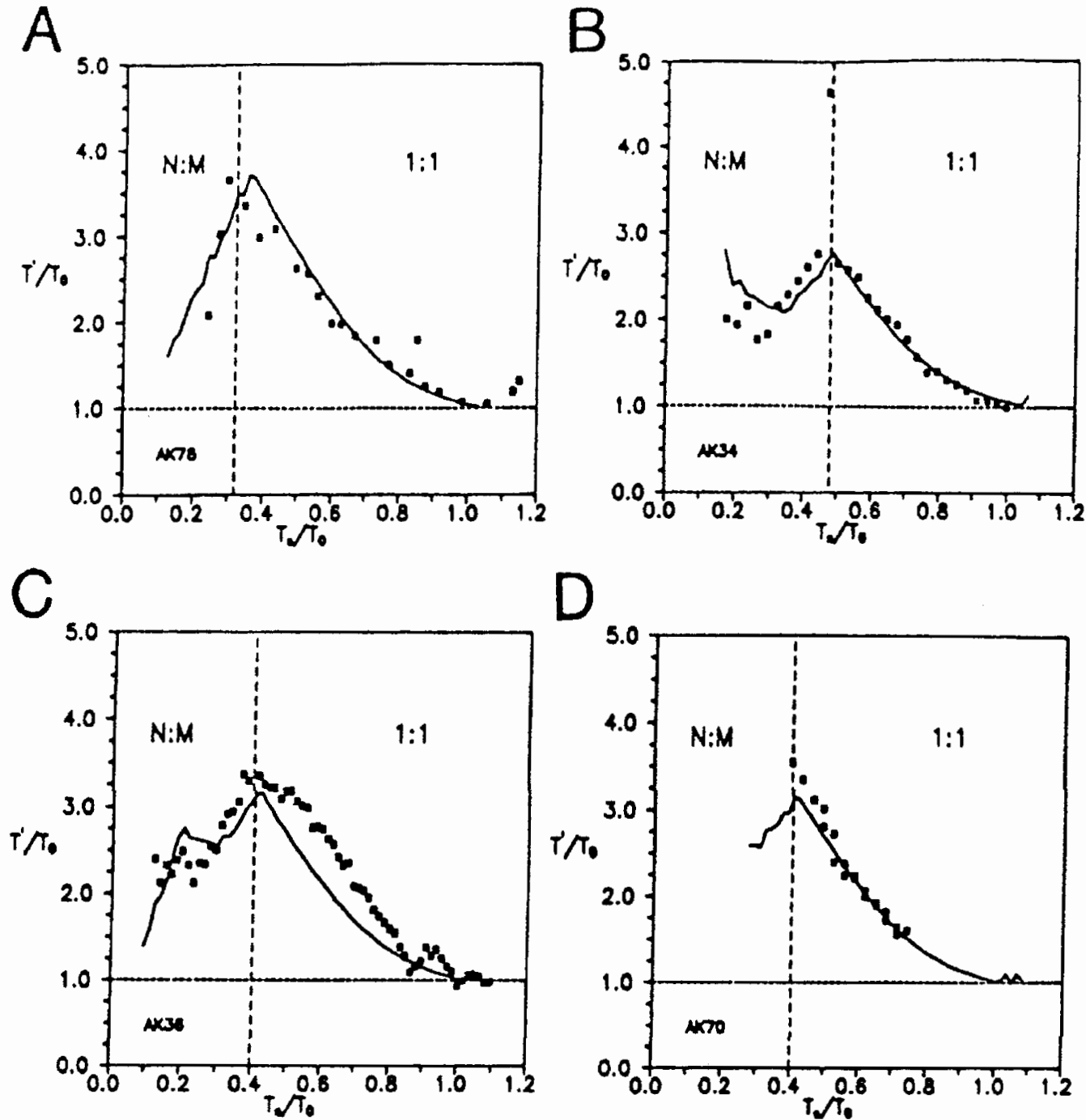

D

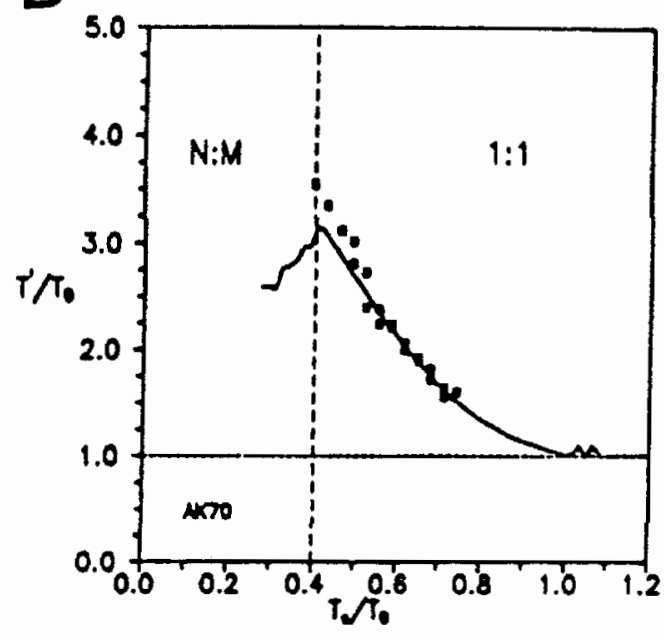

FIGURE 7 Composite picture of the first cycle length following 50 stimuli as a function of the period of the stimulation, for four different aggregates (filled symbols), superimposed on the simulation of the theoretical model described in Kunysz et al. (1995) (solid line). Adapted from Kunysz et al. (1995), by permission.
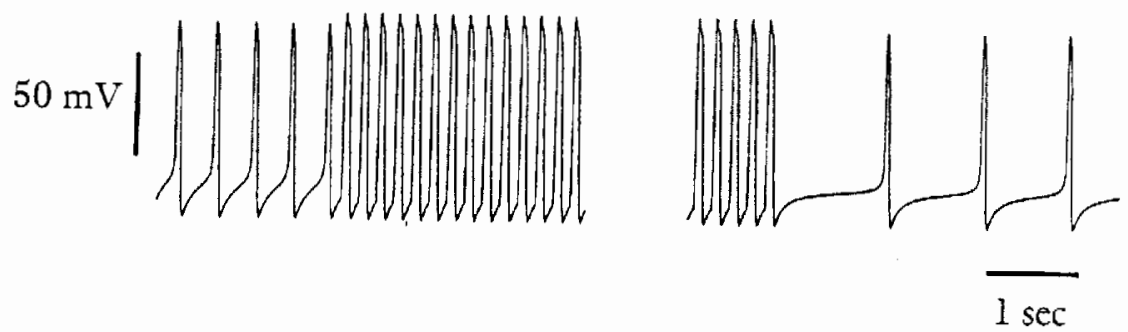

FIGURE 8 Simulation of overdrive suppression in the Shrier-Clay ionic model modified to include a simplified sodium -potassium pump term (see Kunysz et al. . 1997). The control cycle length is $400 \mathrm{msec}$. A few spontaneous action potentials appear on the left. For clarity, not all action potentials are shown. The application of 100 high intensity stimuli $\left(t_{\mathrm{s}}=0.5 T_{0}\right.$, amplitude $=90 \mathrm{nA}, 1: 1$ entrainment) results in a transient three-fold prolongation in cycle length. Compare with Figure 6. 
of the excitability properties of the preparation, as described by the phase resetting curve. In their theoretical model, in the form of a two-dimensional difference equation, each premature action potential contributes to overdrive suppression by decreasing the slope of diastolic depolarization (phase 4). The contribution of each action potential decays exponentially with time; after cessation of stimulation the cycle length returns to control. The effect of the changes in the intrinsic cycle length on phase resetting are implemented by assuming that, at any time, the phase of the stimulus must be rescaled to the instantaneous intrinsic cycle length. Similarly, the amount of delay or shortening of the cycle length due to phase resetting depends upon the intrinsic beat rate.

This model has direct implications for analyzing the effects of long stimulation trains delivered to the aggregates. Although the phase locked patterns are stable, there is often an evolution of rhythms during stimulation. For example, at high stimulus intensities, rapid stimulation may initially result in 1:1 entrainment which finally (gradually or abruptly) evolves into a 2:1 rhythm. With stimulation at high frequencies, the initial rhythm typically evolves towards higher degree of block (Zeng et al., 1991).

In further experimental studies from our group (Kunysz et al., 1995), it was determined that: (1) although the buildup of overdrive suppression is an increasing function of the number of stimuli applied, it shows a tendency to saturate at relatively fast stimulation frequencies, but increases indefinitely (as long as 1:1 entrainment is maintained) at very high frequencies; (2) action potential frequency is the major determinant of overdrive suppression; (3) overdrive suppression and underdrive acceleration (transient increase in beat rate following stimulation at a rate slower than control) share a common mechanism; (4) overdrive suppression is primarily responsible for the evolution of rhythms observed experimentally; (5) during overdrive suppression, the slope of diastolic depolarization never becomes negative.

Kunysz et al. (1995) developed a model of overdrive suppression based on a modification of the
FitzHugh-Nagumo equation. Overdrive suppression was incorporated by means of a ordinary differential equation that describes the changes in internal sodium levels due to the interplay between the rate-dependent influx of sodium during the upstroke phase of the action potentials and the extrusion of sodium ions by an internal sodium-sensitive electrogenic sodium-potassium pump. Full activation of the sodium-potassium pump decreases the slope of diastolic depolarization to zero. The parameters of the model were set based on the qualitative experimental data. The best agreement with experimental data was obtained by assuming that, under control conditions, the sodium-potassium pump was operating at $60 \%$ of its maximum capacity.

Figure 7 is a composite picture of the first normalized cycle length following 50 stimuli as a function of the period of stimulation (normalized to control), for four different aggregates, superimposed on numerical simulations of the theoretical model. With 1:1 phase locking, the duration of the postdrive pause is inversely proportional to the period of stimulation. At higher stimulation frequencies ( $N: M$ entrainment, $N>M$ ), overdrive suppression decreases as a result of dropped beats, giving rise to the characteristic 'peaking phenomenon'. The 'peaking phenomenon' is thus associated with sudden changes in action potential frequency due to the transition between $N: M$ and $N^{\prime}: M^{\prime}$ phase locking, where $N / M \leq N^{\prime} / N^{\prime}$. This behavior is consistent with the numerical simulations. However, the application of this theoretical model to a wider body of experimental data is limited by deficiencies in reproducing the proper phase resetting behavior (Kunysz et al., 1995).

To overcome these deficiencies Kunysz et al. (1997) have included terms modeling overdrive suppression in the Shrier-Clay ionic model. This model shows good agreement with qualitative features of overdrive suppression (Figure 8). A sharp challenge to the model is to carry our repeated stimulation at a fixed delay following the onset of an action potential. Experimental studies show complex rhythms some of whose features are well reproduced by the ionic model. 


\section{CONCLUSIONS}

We have summarized research from our group concerning the dynamics observed during stimulation of spontaneously beating heart cell aggregates using a variety of different experimental protocols. Although a simplified nonlinear iterative model, equation (1), gives good agreement with a broad range of effects observed during periodic stimulation, it is not adequate to reproduce the timedependent effects such as overdrive suppression, that are induced during prolonged electrical stimulation. Similarly, although the Clay-Shrier ionic model as originally formulated enables us to derive insight into the mechanisms of phase resetting, it did not contain time-dependent terms that could lead to overdrive suppression. We have shown how combination of experimental studies with nonlinear and ionic modelling approaches can lead to insight into the origin of complex rhythms observed experimentally.

Similar time-dependent phenomena have been observed in a number of different preparations. For example, these include depressed conduction at Purkinje-muscle junctions (Gilmour et al., 1987), rate dependent changes in action potential duration (Kawano and Hiraoka, 1991), and atrio-ventricular nodal fatigue (Talajic et al., 1991; Sun et al., 1995). Despite the variety of possible underlying mechanisms, these experimental observations can be explained by hypothesizing that excitability is a function of previous activity or stimulation history. Excitability is depressed by more increased activity and enhanced by reduced activity. However, there is still a gap between the phenomenological observations and theoretical modeling of these effects. The methods applied here should have broad applicability to a variety of other systems.

Ionic models and nonlinear models are complementary. Ionic models successfully reproduce the action potential based on detailed studies of the underlying ionic currents (patch-clamp, pharmacological studies). Nonlinear models capture the essence of the dynamics without giving details of the mechanisms. They can often be used as a preliminary step towards the development of more realistic ionic models. Conversely, ionic models are seldom tested for comparison with their nonlinear counterparts, as well as with experimental data that does not originate from patch-clamp experiments. We challenge individuals working on the development of ionic models to test their models by exercising them against experimentally observed dynamics obtained during a broad range of different stimulation protocols.

\section{Acknowledgments}

We thank the Quebec Heart and Stroke Foundation, the Natural Sciences Engineering Research Council, and the Fonds pour la Formation de Chercheurs et l'Aide à la Recherche for their continuing support of the research summarized here.

\section{References}

Beaumont, J., Michaels, D. C., Delmar, M., Davidenko, J. and Jalife, J. (1995) A model study of changes in excitability of ventricular muscle cells: inhibition, facilitation, and hysteresis. Am. J. Physiol., 268, H1181-HI194.

Chialvo, D. R. (1990) Toward very simple generic models of excitable cells. Order and chaos in cardiac tissues. Facts and conjectures. Ann. N.Y. Acad. Sci, 591, 351-366.

Clay, J. R., Guevara, M. R. and Shrier, A. (1984) Phase resetting of the rhythmic activity of embryonic chick heart cell aggregates: Experiment and theory. Biophys. J., 45, 699-714.

Clay, J. R., Brochu, R. M. and Shrier, A. (1990) Phase resetting of embryonic atrial chick heart cell aggregates. Experiment and theory. Biophys. J., 58, 609-621.

Courtney, K. R. and Sokolove, P. G. (1979) Importance of electrogenic sodium pump in normal and overdriven sinoatrial pacemaker. J. Molec. Cell. Cardiol, 11, 787.

DeHaan, R. L. (1967) Regulation of spontaneous activity and growth of embryonic chick heart cells in tissue culture. Devel. Biol., 16, 216-249.

Dexter, F., Levy, M. N. and Rudy, Y. (1989) Mathematical model of the changes in heart rate elicited by vagal stimulation. Circ. Res., 65(5), 1330-1339.

Dokos, S., Celler, B. G. and Lovell, N. H. (1993) Modification of DiFrancesco-Noble equations to simulate the effects of vagal stimulation on in vivo mammalian sinoatrial node electrical activity. Ann. Biomed. Eng., 21(4), 321-335.

Gilmour, R. F., Davis, J. R. and Zipes, D. P. (1987) Overdrive suppression of conduction at the canine Purkinje-muscle junction. Circulation, 76, 1388-1396.

Glass, L., Guevara, M. R., Bélair, J. and Shrier, A (1984) Global bifurcations of a periodically forced biological oscillator. Phys. Rev. A, 29, 1348-1357.

Glass, L., Shrier, A. and Bélair, J. (1986) Chaotic cardiac dynamics, in A. Holden, (ed.) Chaos, (Princeton University Press, Princeton.), pp. 235-256, 
Glass, L. and Mackey, M. (1988) From Clocks to Chaos: The Rhythms of Life, (Princeton University Press, Princeton).

Greenberg, Y. J. and Vassalle, M. (1990) On the mechanism of overdrive suppression of automaticity in the guinea pig sinoatrial node. J. Electrocardiol., 23, 53-67.

Guevara, M. R., Glass, L. and Shrier, A. (1981) Phase-locking, period-doubling bifurcations and irregular dynamics in periodically stimulated cardiac cells. Science, 214, 1350-1353.

Guevara, M. R. and Glass, L. (1982) Phase-locking, perioddoubling, and chaos in a mathematical model of a periodically driven oscillator: A theory for the entrainment of biological oscillators and the generation of cardiac arrhythmias. J. Math Biol, 14, 1-23.

Guevara, M. R., Glass, L. and Shrier, A. (1986) Phase resetting of spontaneously beating embryonic ventricular heart cell aggregates. Am. J. Physiol., 251, H1298-H1305.

Kawano, S. and Hiraoka, M. (1991) Transient outward currents and action potential alterations in rabbit ventricular myocytes. J. Molec. Cell. Cardiol., 23, 681-693.

Kowtha, V. C., Kunysz, A., Clay, J. R., Glass, L. and Shrier, A (1994) Ionic mechanism and nonlinear dynamics of embryonic chick heart cell aggregates. Prog. Biophys. Molec. Biol, , 61, 255-281.

Kunysz, A. M., Glass, L. and Shrier, A. (1995) Overdrive suppression of spontaneously beating embryonic chick heart cell aggregates: experiment and theory. Am. J. Physiol., 269, H1153-H1164.

Kunysz, A. M., Shrier, A. and Glass, L. (1997) Bursting behavior during fixed delay stimulation of chick heart cell aggregates. Am. J. Physiol., 273, (cell Physiology 42), C331-C346.

Liu, Y., Zeng, W., Delmar, M. and Jalife, J. (1993) Ionic mechanisms of electrotonic inhibition and concealed conduction in rabbit atrioventricular myocytes. Circulation, 88(1), $1634-1646$.

Luo, C. H. and Rudy, Y. (1991) A model of the ventricular cardiac action potential. Depolarization, repolarization, and their interaction. Circ. Res., 68(6), 1501-1526.

McAllister, R. E., Noble, D. and Tsien, R. W. (1975) Reconstruction of the electrical activity of cardiac Purkinje fibres. J. Physiol. (Lond.), 251, 1-53.

Moore, J. W. and Ramon, F. (1974) On numerical integration of the Hodgkin and Huxley equations for a membrane action potential. J. Theor. Biol., 45, 249-273.

Musso, E. and Vassalle, M. (1982) The role of calcium in overdrive suppression of canine cardiac Purkinje fibers. Civc. Res., 51, 167-180.

Noble, D. and Noble, S. J. (1984) A model of sino-atrial node electrical activity based on a modification of the DiFrancesco-Noble (1984) equations. Proc. R. Soc. Lond. B Biol. Sci, B222, 295-304.

Pavlidis, T. (1973) Biological Oscillators: Their Mathematical Analysis, (Academic Press, New York.)
Pelleg, A., Vogel, S., Belardinelli, L. and Sperelakis, N. (1980) Overdrive suppression of automaticity in cultured chick myocardial cells. Am. J. Physiol, 238, H24-H30.

Perkel, D. H., Schulman, J. H. and Bullock, T. H. (1964) Pacemaker neurons: effect of regularly spaced synaptic input. Science, 145, 61-63.

Prinsze, F. J. (1993) Cellular Aspects of Overdrive Suppression in the Sinoatrial Node, $\mathrm{Ph} \mathrm{D}$ Thesis. University of Amsterdam, Amsterdam.

Rudy, Y. and Luo, C. H. (1993) Cellular responses to electrical stimulation: a study using a model of the ventricular cardiac action potential. Adv. Exp. Med. Biol., 346, 79-90.

Rush, S. and Larsen, H. (1978) A practical algorithm for solving dynamic membrane equations. IEEE Trans. Biomed. Eng., 25, 389-392.

Shrier, A. and Clay, J. R. (1986) Repolarization currents in embryonic chick atrial heart cell aggregates. Biophys. J., 50, 861-874.

Sun, J., Amellal, F., Glass, L. and Billette, J. (1995) Alternans and period-doubling bifurcations in atrioventricular nodal conduction. J. Theor. Biol., 173, 79-91.

Talajic, M., Papadatos, D., Villemaire, C., Glass, L. and Nattel, S. (1991) A unified model of AV node conduction explains dynamic changes in Wenckebach periodicity. Circ. Res., 68, $1280-1293$.

van der Pol, B. and van der Mark, J. (1929) The heartbeat considered as a relaxation oscillator, and an electrical model of the heart. Arch. Neerl. Physiol., 14, 418-443.

Vassalle, M. (1970) Electrogenic suppression of automaticity in sheep and dog Purkinje fibers. Circ. Res., 27, 361-377.

Vassalle, M. (1977) The relationship among pacemakers: Overdrive suppression. Circ. Res., 41, 269-277.

Vinet, A. and Roberge, F. A. (1994) Analysis of an iterative difference equation model of the cardiac cell membrane. $J$. Theor. Biol., 170(2), 201-214.

Wilders, R., Jongsma, H. J. and van Ginneken, A. O. G. (1991) Pacemaker activity of the rabbit sinoatrial node. A comparison of mathematical models. Biophys. J., 60, 1202-1216.

Winfree, A. T. (1980) The Geometry of Biological Time, (Springer-Verlag, New York).

Winfree, A. T. (1987) When Time Breaks Down, (Princeton University Press, Princeton).

Zeng, W. Z., Courtemanche, M., Sehn, L., Shrier, A. and Glass, L. (1990) Theoretical computation of phase locking in embryonic chick heart cell aggregates. J. Theor. Biol., 145, 225-244.

Zeng, W. Z., Glass, L. and Shrier, A. (1991) Evolution of rhythms during periodic stimulation of embryonic chick heart cell aggregates. Circ. Res., 69, 1022-1033. 


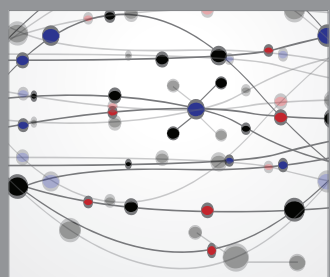

The Scientific World Journal
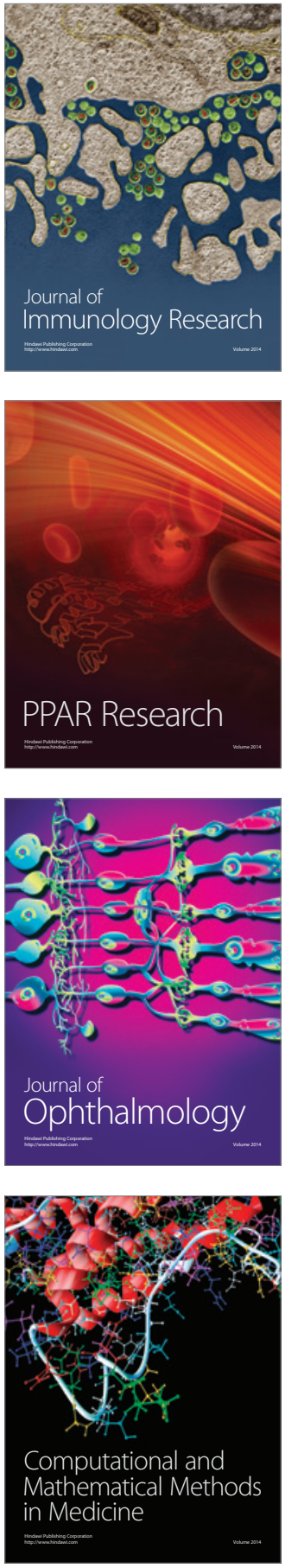

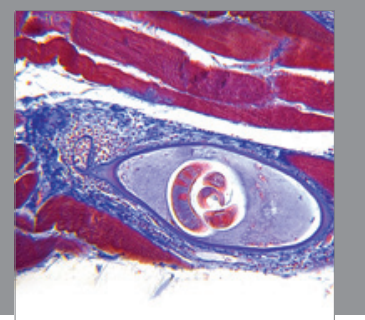

Gastroenterology

Research and Practice
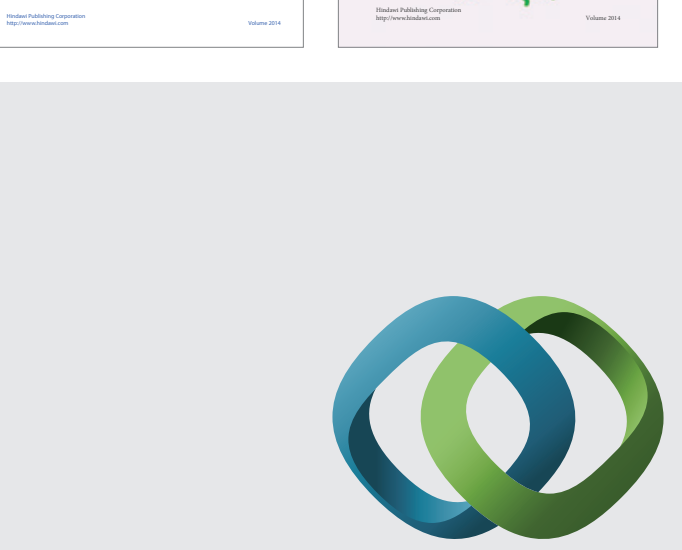

\section{Hindawi}

Submit your manuscripts at

http://www.hindawi.com
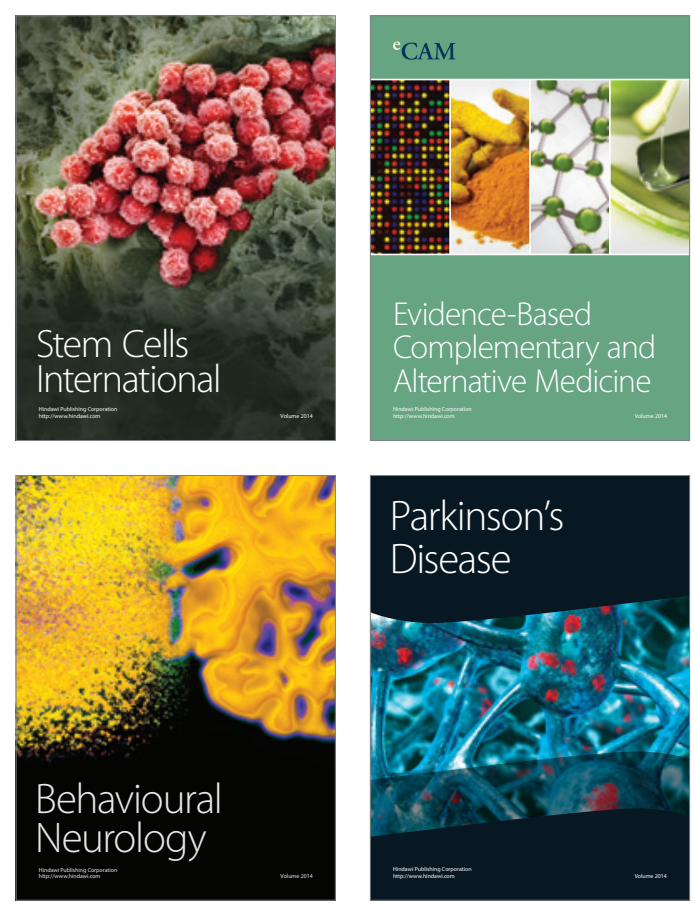

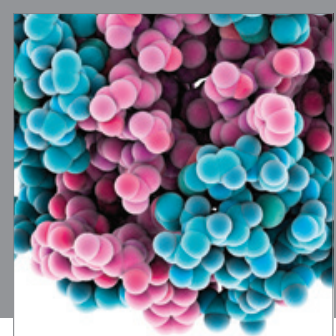

Journal of
Diabetes Research

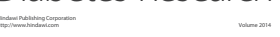

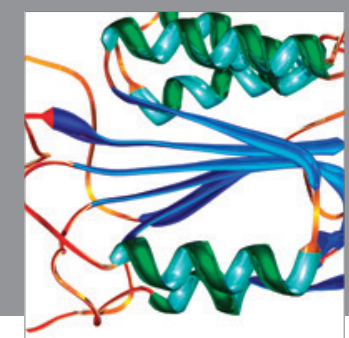

Disease Markers
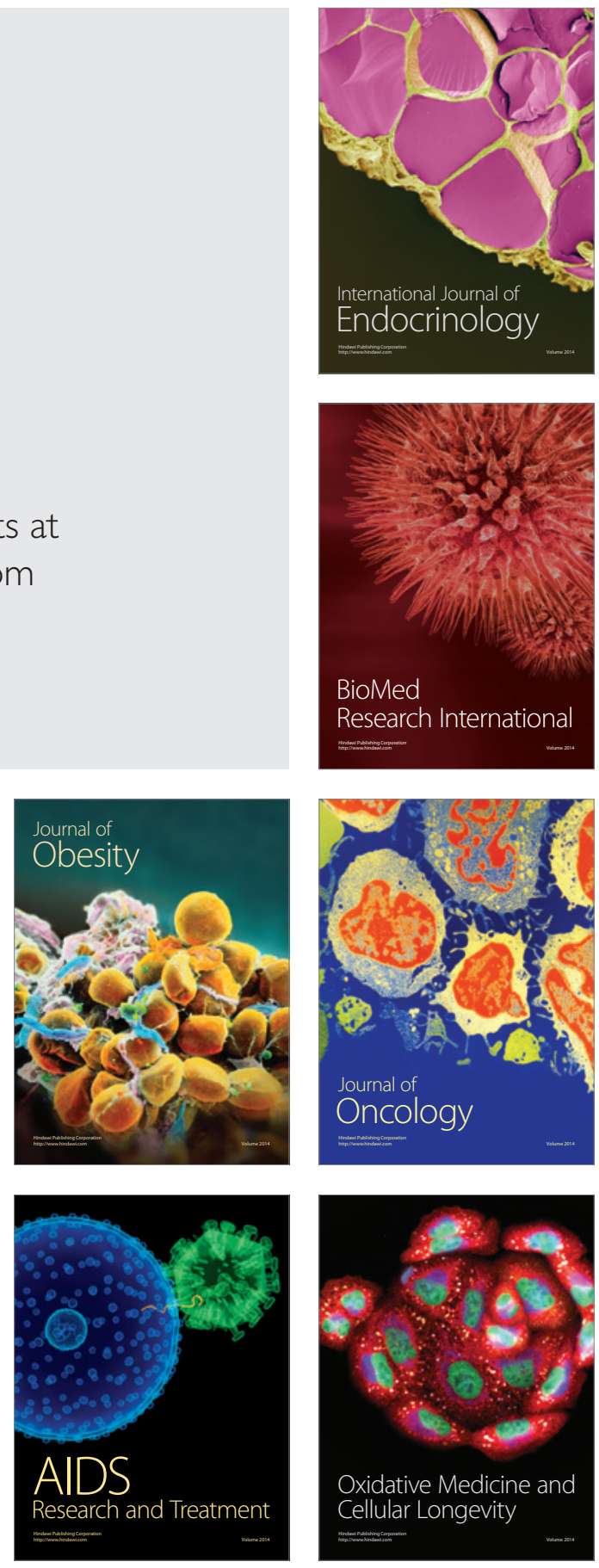